\title{
NATURALEZA, MUERTE Y MAL: NOTAS SOBRE TOMÁS DE AQUINO
}

\author{
José Cercós Soto \\ Profesor de Filosofía. I.B. Huelva
}

Es indudable la atracción que la dilucidación del mal ejerce sobre la filosofía. No, precisamente, por su lejanía sino por ser «algo» con lo que cotidianamente nos topamos. Una enumeración exhaustiva sería, ciertamente, penosa: las catastrofes de Bangla Desh, terremotos, las guerras en distintos puntos de nuestro planeta... 0 , muchio más cerca y no por eso menos malignas, las diversas plagas con las que se enfrenta nuestra sociedad: drogas, terrorismo, corrupción, etc. Es obvio que no nos podemos ocupar de las causas particulares de cada uno de estos problemas, que no es el asunto de la filosofía, sino que nos preocupa la comprensión metafísica del mal. ¿Qué es el mal? Y, sobre todo, ¿cuál es su sentido? Son las preguntas que intentamos responder. Para lo cual vamos a utilizar y exponer el pensamiento de Tomás de Aquino como forma de acercamiento a la cuestión. A la vez nos vamos a centrar no en el mal moral, ${ }^{1}$ sino en el mal natural. Esto, en última instancia, nos conducirá a hablar de la muerte.

\section{EL MAL EN SÍ MISMO}

Indudablemente, para el Aquinate el lugar del mal es, ontológicamente, secundario. Para aclarar el significado de ese carácter secundario hemos de partir del pensamiento de Tomás acerca del ser: como sabemos, es una cuestión de suma importancia en la comprensión de la estructura de la realidad. Porque «ser», en esencia, es Dios -Ipsum esse subsistens-; mientras que lo creado es «ser participado» y, por eso, ya limitado. Como limitado, no puede tener la radical simplicidad de lo divino. Por eso, nos señala Tomás, con profusión, los varios modos del ser. Por un lado, aquél presentado bajo las diez categorías, es decir, el ente estructurado en torno a la substancia y lo que

1 Cfr. Forment, E., "Génesis del mal moral", en A. Lobato (ed.), Littera sensus sententia. Studi in onore del Prof. C.J. Vaansteenkiste, Massimo, Milano 1991, pp. 251-284. 
en ella se da. Por otro, aquel acto intrínseco al ente concreto que «arrastra» a las determinaciones esenciales de ese ente al existir: el actus essendi. Y, por último, el ser mental.

Es en torno a esta clasificación como Sto. Tomás ha de ver ese lugar secundario del mal: el quedar reducido a lo mental: «el ente se dice de dos formas. En una significa la naturaleza de los diez géneros; y así ni el mal ni alguna privación es ente o algo. En la otra, en tanto que responde a la cuestión «si es»; y así, el mal es...». ${ }^{2}$ Es claro que hay «cosas» cuya entidad es nula aparte de su posible consideración intelectual -ser mental-, mientras que en otras a esta consideración se le une su efectiva existencia real. El pensamiento de Tomás en este respecto es muy claro en sus obras -como ya se ha mostrado en la anterior cita-; el mal «es» en el sentido veritativo, mas carece de ser si acudimos a la naturaleza contemplada a través de los diez géneros: «es imposible que el mal signifique algún ser». ${ }^{3}$ Nos encontramos, en consecuencia, ante un «real» defecto de ser. ${ }^{4} 0$, de otro modo, ante una «positiva negatividad». En cuanto que puede ser que el mal no tenga ser real-ciertamente no tiene la misma entidad que una cualidad positiva-, pero realmente el mal es más que la mera constatación del ser mental. Estrictamente, el mal es privación de un bien debido, ${ }^{5}$ del ser debido.

Aclaremos brevemente qué entiende Tomás por «privación». Para que pueda hablarse de «privación» hay dos exigencias: primero, la eliminación o ausencia del hábito opuesto. Segundo, «que la privación propiamente dicha sea sobre un sujeto determinado y un tiempo determinado».6 Si lo aplicamos al mal, todo mal se da en algo, en un momento determinado, al carecer del bien que debería tener. Ahora bien, en la naturaleza, el mal ha de referirse a la falta de alguna cualidad necesaria para la «normalidad» -quando aptus natus est habere- del ser concreto: mal es ser defectuoso? ' La muerte, como último mal, sería ya la privación del sujeto y del tiempo de ese sujeto.

2 De malo, q. 1, a. 1, ad 19. Cfr. A. García Marqués, Necesidad y substancia, EUNSA, Pamplona 1989, pp. 75 ss.: «Ser veritativo y ser extramental».

3 S.th., l, q. 48, a. 1.

4 «Bonum et malum actionis, sicut et ceterarum rerum, attenditur ex plenitudine essendi vel defectu ipsius»: S.th., I-II, q. 18, a. 2.

5 «Así como entendemos por bien la perfección del ser, por mal se entiende la privación de esta perfección. Pero, como la privación propiamente dicha es la privación de un bien debido, que le pertenece en un tiempo y de un modo determinado, es evidente que una cosa es llamada mala porque carece de una perfección que debe tener»: Compendio de Teología, c. 114.

6 In Met., V, lt. 14, n. 967.

7 «Privatio dicitur multipliciter. Uno enim modo quicquid non habet aliquid, potest dici esse privatum; sicut si dicamus lapidem privatum esse, per eo quod non habet visum. Alio modo dicitur privatum solum quod est aptum natum habere, et non habet. Et hoc dupliciter. Uno modo universaliter quando non habet; sicut si dicatur canis privatus visu, quando non habet visum. Alio modo si non habet, quando aptus natus est habere. Unde canis ante nonum diem non dicitur privatus visu. Et iterum hoc diversificatur. Nam uno modo dicitur privatum eo quod non habet aliquo modo determinato, scilicet perfecte et bene sicut cum vocamus caecum eum qui non bene videt. Alio modo quando non habet omnino; sicut dicimus privatum visu, qui omnino visum non habet. Quandoque vero in ratione privationis includitur violentia»: In Met., IX, 1t. 1, n. 1785. Así, el mal comienza por el defecto en algo que se debe tener por naturaleza, ya sea una falta parcial -qui non bene videt-o total -omnino visum non habet-. Tomás afirma en otro lugar que sólo tiene razón de mal la carencia de bien entendida como privación, no como «negatividad»-cfr. S.th., I, q. 48, a. 3-. «Negatividad» que no es sino el primero de los sentidos de privación en el texto de In Met. que acabamos de transcribir; es decir, que algo no tiene mal en cuanto carece de cualquier cosa, sino en la carencia de lo debido. 
En este «optimismo ontológico» que es la cosmovisión tomista, porque el ser, de por sí, es bueno: «todo ente, en cuanto es ente, es bueno». ${ }^{8} \mathrm{El}$ mal tiene el ser de lo que es ser-defectuoso: es como un «hueco» en el que el ser es no como debe ser. $Y$ aunque no se le debe negar realidad, no es todo lo real que podría ser, definidos los niveles de la realidad por la «calidad» del ser.

En conclusión, el carácter ontológicamente secundario del mal exige la bondad del mero hecho de ser, de forma que cualquier privación en este ser -el defecto de lo debido-nos hace concebirlo como mal. Lo cual lleva a afirmar que, ya que el mal se encuentra tan profusamente presente en nuestra vida, existe un rádical déficit de ser: la realidad de lo que hay dista bastante de poseer la perfección debida.

Aquí es interesante dejar constancia de que Tomás afirma claramente, como ya hemos comentado, la doble referencia del mal: in rebus, in moralibus. Según se considere uno u otro, quién pueda ser sujeto del mal variará. Pues el primer respecto -in rebus- se produce en seres distintos a los del segundo: «aun cuando la ausencia del mal de la naturaleza sea algo común a todas las cosas incorruptibles, el estar exento, por necesidad de su naturaleza, del mal de culpa, del que sólo es capaz la naturaleza racional, sólo es propio de Dios». ${ }^{9}$ Así, todo ser inteligente, excepto Dios, puede llegar a ser culpable, ${ }^{10}$ mas sólo lo definido esencialmente por la materialidad es susceptible del mal natural. De aquí podría deducirse, en general, que el fundamento metafísico de la posibilidad de mal se encuentra en la escisión propia de toda criatura entre su ser y su poder ser. Hay posibilidad de mal en todo en lo que es imposible la identificación de su esencia con su existencia: en lo insuficiente entitativamente.

$\mathrm{El}$ «optimismo ontológico» del Aquinate, evidentemente compatible con el hecho de que el ser creado sea insuficiente, imposibilita la existencia de algo malo por naturaleza; lo real existente lo es por el actus essendi que «arrastra» las notas esenciales a su efectivo ser. Y, si existe y mientras es, no puede cesar en él. Por lo que -excepto en el mal de la muerte en el que se da el acabar del existir- el mal se hace «efectivo» en el orden estructurado sobre el núcleo esencial-existencial: «así como la realidad natural no cesa en el ser que le compete según su forma, en cambio puede cesar en algunas cosas accidentales o consecuentes...>. ${ }^{11}$ El mal -excepto la muerte- es un déficit ${ }^{12}$ o falta de lo debido en el ser accidental: por eso, si el ente como tal

8 S.th., I, q. 5, a. 3.

9 Comp. de Teol., c. 120.

10 Recordemos que el mal de culpa es el defecto en las acciones voluntarias.

11 S.th., I, q. 17, a. 3.

12 ¿Puede darse así explicación de la abrumadora «positividad» del mal que experimentamos? El mal, cierto, es ausencia de bien; pero esa ausencia se traduce realmente por el mal presente, que «es» ahí. Por otro lado, no habría dolor sin ese efectivo estar presente del mal: «ad dolorem duo requiruntur; scilicet coniuctio alicuius mali (quod ea ratione est malum, quia privat aliquod bonum); et perceptio huiusmodi coniuctionis»: S.th., I-II, q. 35, a. 1. ¿No podría ser que -en la naturaleza-no hay ni bien ni mal, sino sólo ser? Quizás en este sentido pueda entenderse la afirmación de que «el mal no es existente» -S.th., I, q. 48, a. 1-. Sólo hay el ser: el mal exige la interpretación del ser humano de acuerdo con sus conveniencias. ¿Y que es el hombre el que «pone» la bondad o la maldad donde de suyo no existe? ¿Es bueno o malo que el águila capture su presa o, como debe ser así, no es ni bueno ni malo? ¿Es en sí mismo malo un ciclón o la erupción de un volcán? No, sino sólo en cuanto daña al hombre -quoad nos-. Por eso, dice Sto. Tomás que el bien y el mal propiamente, pertenecen a la moral: no son categorías «naturales». El mal in rebus no es sino el estar-ahí de lo que no debería ser-ahí en cuanto contraviene la «normalidad» natural. Mas en lo material, ¿qué es normalidad? 
es bueno, el mal se dará siempre sobre el bien y en aquello en lo que está en potencia a un bien determinado. ${ }^{13}$

Por consiguiente, y contemplado en sí mismo, en el mal nos encontramos con la privación de un bien que debe ser, por tanto con una falta de ser, un ser disminuido. La muerte no es sino el mal natural por excelencia: el «último» mal natural consistente en la privación del existir. Mas si mal supone contravenir la «normalidad-natural», y a la materia, de suyo, le es natural la «descomposición», parecería que no podría entenderse la muerte e incluso otra serie de defectos como «anormales». Por eso, Santo Tomás discute la exclusiva naturalidad de la muerte.

Así, en De malo ${ }^{14}$ comienza distinguiendo dos sentidos de naturaleza. $1^{\circ}$ Lo que llamamos propiamente natural, como los «cuerpos naturales». 20. Aquello que es consecuencia del ser natural; esto, a su vez, puede decirse de dos formas, pues es distinto lo natural según la materia y según la forma. a) Según la forma es natural que el fuego caliente. b) Según la materia, es natural que el agua pueda calentarse. Lo que sigue a la materia también puede entenderse de dos modos. i) en cuanto concuerda con la forma: así, en el ser humano, es natural la composición del cuerpo que posibilita la percepción que será el punto de arranque del proceso cognoscitivo. ii) en cuanto repugna a la forma, la materia es corruptible.

De este modo, la muerte sólo es natural en cuanto la corrupción es exigida por la naturaleza de la materia, pues lo conveniente a la forma es la inmortalidad. ${ }^{15} \mathrm{La}$ forma, a la vez, se sabe inmersa en la materialidad; luego su «inmortalidad» se sabe mediatizada por la naturalidad de la muerte y de la imperfección de lo material. «Formalmente», así, puede juzgarse como mal aquello que «materialmente» es normal y, en consecuencia, ni bueno ni malo. Mas el hombre es un todo, con una «normalidad-natural» y un «proceso natural» que debe cumplir. El defecto en su naturaleza es mal. Pero la muerte se considerará mal no si se toma al ser humano tal y como es, sino «como fue».

Así, la «materialidad» no es el único ni el más importante de los factores que, para Santo Tomás, provoca la muerte: lo veremos en las causas.

13 «Quia verum et bonum communia sunt, et convertuntur cum ente: unde sicut omnis privatio fundatur in subiecto quod est ens, ita omne malum fundatur in aliquo bono»: S.th., I, q. 17, a. 4, ad 2. «No todo bien puede ser sujeto del mal, sino solamente el bien que es potencia respecto de alguna perfección de la que puede estar privado. Por tanto, en las cosas que son solamente acto, $o$ en las que el acto no puede estar separado de la potencia, no puede existir el mal en tal sentido»: Comp. de Teol., c. 117.

14 De malo, q. 4, a. 5.

15 En S.th., I-II, q. 85, a. 6, Tomás distingue entre «naturaleza universal» y «naturaleza particular». La naturaleza particular tiende a la persistencia en su estado. Mientras que la naturaleza universal es el principio que se propone el orden del universo y su bien, para lo cual se necesita la generación y corrupción de las cosas. 


\section{DE LAS CAUSAS DEL MAL Y LA MUERTE}

Mas, ¿puede el déficit de ser tener causas determinadas? Obviamos, por el momento, la cuestión de Dios y el mal y, en consecuencia, si Dios como causa de todo también lo es del mal.

Dos son las preocupaciones de Tomás a la hora de intentar concretar el asunto de las causas del mal. En primer lugar, que el mal, en cuanto arrastra ese vacío de ser o plenitud de ser indebido, no puede tener una causa per se. Tres son las razones que nos da en la cuestión disputada De malo: a) que nadie persigue el mal como un fin en sí mismo; b) que el causar, como acto, tiene razón de ser y, en consecuencia, de bien; c) que la causa per se persigue y se ordena a un fin determinado; mas lo que se realiza según un orden no puede tener razón de mal. Así, en opinión del Aquinate, ningún elemento de una acción puede perseguir directamente la consecución del mal: ni la finalidad pretendida, ni el modo determinado del actuar, ni el actuar como tal actuar. El ser sólo puede «apetecer» el ser, no el no-ser; por eso, el no-ser sólo puede ser algo que accidentalmente acontece..$^{16}$

En la segunda preocupación, se trataría de precisar la dimensión de la causalidad del mal a través de las cuatro clases de causas. Desde lo anteriormente dicho parece absolutamente claro que-ya que el mal «no es existente» ${ }^{17}$ ni es perseguido como tal por nadie-no puede tener causa final ni formal. En cambio, ya que el mal siempre es producido por algo, tiene causa eficiente -aunque accidentalmente-; y tiene causa material, pues ya hemos dicho que el mal -en cuanto carece de «densidad» ontológica- siempre ha de darse en algo que es y, en tanto que es, es bueno.

Hasta ahora hemos intentado dejar claro que todo defecto en la realidad natural es debido a la materialidad, que es una de las dimensiones esenciales del ser humano. Mas esta materialidad no es la única causa, porque piensa Tomás que tiene tanta o más importancia el pecado original. Porque antes de que se separe de Dios por el pecado, el hombre en el «estado de inocencia» era inmortal. No por la materia ni por la forma, sino por una fuerza divina y sobrenatural que le protegía de toda corrupción. ${ }^{18}$ Por consiguiente, el carácter defectuoso y mortal del ser humano está provocado tanto por la materialidad como por la pena impuesta a raíz del pecado original. Y es que una vez sustraída la «justicia original», el hombre queda sometido a la naturaleza de la materia. Así, «la causa de la muerte y de los demás defectos de la naturaleza humana es doble. La primera remota, que se toma de los principios materiales del cuerpo humano, en cuanto se compone de contrarios. Mas esta causa quedaba impedida por la justicia original. $Y$, por eso, la causa próxima de la muerte y de los otros defectos es el pecado,

16 Que el «no-ser acontezca» se entiende dentro del marco de la discusión, en la que ya hemos afirmado la «realidad» del mal. Por otro lado, ¿qué pensar de aquellas acciones en las que se busca un beneficio propio que se sabe va indisolublemente acompañado del mal ajeno e, incluso, del mal propio? ¿No es, casi, buscar el mal como tal?

17 S.th., I, q. 48, a. 1.

18 «Nom enim corpus erat indissolubile per aliquem immortalitatis vigoris in eo existens; sed inerat animae vis quaedam supernaturaliter divinitus data, per quam poterat corpus ab omni corruptione praeservare»: S.th., I, q. 97 , a. 1 . 
por el que se elimina la justicia original». ${ }^{19}$ Es así que, contemplando cómo fue el hombre, su pecado -alejamiento de Dios- provoca todos los males naturales propios de la imperfección de su ser.

Hasta aquí, este breve repaso del pensamiento de Tomás de Aquino acerca del mal y la muerte respecto a sus causas. Sin duda, es falta del ser que debe ser; mas esa falta ha de ser comprendida como positiva falta: como realidad. En caso contrario, no podría considerarse el dolor como algo que necesita, para que se produzca, de un mal y de la conciencia de la posesión de ese mal. Hay que afirmar la realidad de la privación más allá del mero ser mental. Pero aún nos quedan algunas cuestiones importantes: ¿qué sentido tiene el mal? Y, sobre todo, ¿qué tiene que ver Dios con el mal?

\section{EL SENTIDO: EL LUGAR DEL MAL Y DE LA MUERTE}

Curiosamente, para Tomás, ésta sería una cuestión menor. Dedica artículos a dilucidar la naturaleza del mal y sus causas, pero sobre el asunto de su sentido no se extiende nunca en demasía. Mas, sin duda, es una cuestión interesante; pues, entre otras cosas, suscita la relación de un Dios, que es suma bondad, con las evidentes desgracias que atraviesan el mundo.

Es así, considerando la totalidad de cosas que hay, como el Aquinate afirma el sentido del mal: «la perfección de todo exige que haya ciertas cosas en las que no pueda darse el mal y otras que pueden sufrir los defectos del mal según su naturaleza». ${ }^{20} \mathrm{El}$ universo está construido a la manera de un sistema graduado en el que se dan todos los posibles niveles de perfección: desde el puro y perfectísimo acto del «constructor»-Dios-, hasta la absoluta potencia de la materia. En esta diversidad, como ya hemos señalado anteriormente, los entes definidos por su materialidad son sujetos del mal natural: cuya definitiva expresión será la muerte. Mientras que todos los definidos por la inteligencia, sean o no materiales, son capaces del mal en sus actos voluntarios. Por consiguiente, la construcción del universo incluye la posibilidad de la realidad del mal, de forma que un universo en el que no hubiese mal no podría considerarse como «completo»:21 «Dios y la naturaleza realizan lo que es mejor para el conjunto; mas no lo que es mejor para cada una de las partes, sino en cuanto se ordenan al todo... Por otra parte, este mismo todo que es el conjunto de las criaturas, es mejor y más perfecto, si en él hay algunos que pueden apartarse del bien, que a veces se apartan sin que Dios lo impida».22

19 S.th., III, q. 14, a. 3, ad 2.

20 Comp. de Teol., c. 283. «Perfectio universi requirit inaequalitatem esse in rebus... Sicut igitur perfectio universitatis rerum requirit ut non solum sint entia incorruptibilia, sed etiam corruptibilia; ita perfectio universi requirit ut sint quaedam quae a bonitate deficere possint...»: S.th., I, q. 48, a. 2 .

21 «Optimi agentis est producere totum effectum suum optimum: non tamen quod quamlibet partem totius faciat optimam simpliciter, sed optimam secundum proportionem ad totum»: S.th., I, q. 47, a. 2, ad 1.

22 S.th., I, q. 48, a. 2 , ad 3. 
Por tanto, si no hubiera mal el universo no estaría completo; mas no porque el mal pertenezca a la perfección del universo, sino porque si no existiese «muchos bienes desaparecerían igualmente». ${ }^{23}$ Así, el mal es necesario para el orden del universo, mas no porque éste para su estar completo requiera la falta de ser, sino porque ese mal es la oportunidad para la existencia de otro bien, «así, no se genera el fuego si no se corrompe el aire; ni se conserva la vida del león, si no mata al asno; ni también se alaba la justicia reclamada, si no hubiera injusticia». ${ }^{24}$

Es evidente que sólo es admisible la afirmación de que el mal ha de servir para poder extraer de él algo bueno en cuanto comprende el hacer humano en la vida bajo la noción de «prueba»: antes de gozar de la gloria divina hemos de soportar la pasión. ${ }^{25}$ Así, la muerte se encontraría en la frontera de bien y mal. Por cuanto, naturalmente, se tiende a la conservación del ser; mas, a la vez, la beatitud sólo será tal en la visión de Dios; tras la muerte.

Esas son las dos razones fundamentales que Tomás nos ofrece sobre el sentido del mal. No hay duda de que puede llegar a parecer insuficiente: ¿Cómo decirle al que tiene una enfermedad incurable que el sentido de su dolor - de su mal- es la oportunidad de alcanzar algún bien? ¿ $\mathrm{O}$ a aquellos padres cuyo hijo no es normal? Afirmar la ineludibilidad del mal en el universo es de sentido común. Mas, ¿es imposible un universo sin mal? Ciertamente, mientras haya entes definidos por su materialidad, éstos no podrán substraerse a la corrupción y por tanto al mal. El mal está ligado a nuestra naturaleza material ${ }^{26}$ y a nuestro juicio y valoración de hechos naturales. Habría que indagar, en consecuencia, el sentido de la existencia de la materialidad, que provoca la imperfección de los seres naturales y, en general, el sentido de la escisión ontológica de lo existente. Porque, sin duda, ésta es la razón del mal: la diferencia, frente a la unidad, que fundamenta la potencialidad en los distintos entes, al no ser puro acto.

En la mente de Tomás de Aquino, todo este desarrollo conduce al papel de Dios en el mundo en relación al mal. Porque Dios es el último fundamento de toda existencia, la explicación del ser de lo limitado ha de tomarlo como referencia.

\section{DIOS. CONCLUSIÓN}

«Me doy cuenta una vez más de esto: para cada uno de nosotros, a cada instante, lo peor, lo que consideramos lo peor, es posible: ninguna garantía objetiva. Esto tiene que poder

23 Comp. de Teol., c. 283.

24 S.th., I, q. 48, a. 2, ad 3. «Hoc ergo ad infinitatem Dei bonitatem pertinet, ut esse permittat mala, et ex eis eliciat boni»: ibid., q. 3, a. 1, ad 1.

25 «Oportet enim quod ad immortalitatem et impassibilitatem gloriae, quae in Christo inchoata est, et per Christum nobis acquisita, perveniamus conformati prius passionibus eius»: S.th., I-II, q. 85, a. 5, ad 2.

26 En este sentido, si juzgamos algo malo desde el pensar del bien-privado, es coherente definir el mal como «todo lo que no sea vida eterna»: A. Segura, Emmanuel. Principia Philosophica, Encuentro, Madrid 1982, p. 369. Aunque, eternamente, el ser dotado de voluntad y razón podrá obrar mal moralmente. 
conciliarse con la idea de Dios y de un Dios Todopoderoso. Ahora bien, el hecho de que lo peor sea posible, ¿no prueba la debilidad infinita de Dios? Entre esa debilidad infinita y este poder infinito parece que se opera una unión misteriosa, una coincidencia más allá del orden de las causas». ${ }^{27}$ En este apunte de su Diario metafísico, Marcel hace surgir la paradoja de cómo pensar un Dios infinitamente bueno a la vez que somos conscientes del mal presente en el mundo. Ciertamente, es una cuestión que también se le presentó al Aquinate y desde un doble punto de vista. Porque, por un lado, Dios es causa primera de todas las cosas. El ser de cada cosa depende de Dios de forma que se crea un vínculo, por otro lado, permanente: «igual que nada puede ser que no sea creado por Dios, así nada puede ser que no se someta a su gobierno». ${ }^{28}$ Así pues, siendo Dios primera causa de todo y Aquél que conoce el curso de todo devenir, ¿no será el culpable de todo el mal que acontece? La respuesta del Aquinate diferencia entre las dos clases de mal señaladas: si los agentes voluntarios obran libremente, no puede ser Dios el responsable de esa acción, aunque sepa que ha de producirse. En cambio, considera que el mal que es la corrupción de las cosas sí se reduce a Él como a su causa. ${ }^{29}$ Lo cual se hace evidente si no olvidamos que la muerte tenía una doble causa. La secundaria era la naturalidad de la corrupción de lo material. La más próxima y primera, el que Dios elimina, debido al pecado, ese estado original de inmortalidad poseído por el hombre. Ahora bien, esto ha de matizarse. Porque si Dios es ipsum esse per se subsistens y perfectísimo; y hemos llegado a la conclusión de que el mal es defecto del ser debido; Dios, en consecuencia, no puede ser el «directo» autor del mal. Sto. Tomás, aquí, acude a dos instancias: en primer lugar, que Dios busca la perfección del todo, del Universo; y la perfección de esta totalidad exige la presencia de todos los grados de perfección: desde el mayor hasta el menor. Lo que concluye en que, en aquellos seres cuyo grado de perfección imposibilita su total actualidad, necesariamente han de estar sometidos, al menos, al mal de la corrupción. Pero del mal puede surgir algún bien: por eso Dios permite cierto mal. ${ }^{30}$ Permisividad que, ontológicamente, se traduce en que Dios no puede causar el mal en cuanto tal-lo no ser-si no el bien que a él aparece unido. ${ }^{31} \mathrm{Se}$ explica, así, el mal en referencia a Dios como algo, que si es inevitable porque han de existir seres actopotenciales, seres que en sí mismos son buenos; como algo, decimos, que en sí puede ser punto de partida para «extraer» algo bueno. El mal como excusa para la creación de nuevos bienes: es, para Tomás, el bien del mal.

27 Marcel, G., Diario Metafísico, Guadarrama, Madrid 1969, p. 176.

28 S.th., I, q. 103, a. 5.

29 «Malum quod in defectu actionis consistit, vel quod ex defectu agentis causatur, non reducitur in Deum sicut in causam. Sed malum quod in corruptione rerum aliquarum consistit, reducitur in Deum sicut in causam»: Ibid., q. 49, a. 2.

30 «Multa bona tollerentur, si Deus nullum malum permitteret esse»: Ibid., q. 48, a. 2 , ad 3.

31 «El mal que acontece en las cosas, en cuanto que tiene ser, una especie o una naturaleza cualquiera, se refiere a Dios como a la causa, porque no puede darse el mal sino en el bien... En cuanto a lo que tiene de defectuoso, el mal se refiere a la causa inferior defectible. Por consiguiente, aunque Dios sea la causa universal de todo, no es la causa de los males en cuanto que son males, sino la causa de todo el bien que está unido a tales males»: Comp. de Teol., c. 141. 
Pero esa inevitabilidad evidente es lo que funda la duda, con la que comenzaba este apartado en palabras de Marcel, sobre la verdadera naturaleza divina. ${ }^{32}$

Porque no parece razón suficiente del «ser» del mal la insuficiencia ontológica de determinadas partes del universo en aras de una pretendida perfección del todo. Porque, en última instancia, todo se resuelve en la cuestión de la limitación: ¿por qué va a ser más perfecto un universo en el que existen seres limitados con su consiguiente dosis de mal, dolor y sufrimiento? ¿Cuál es el sentido de la limitación? Porque, filosóficamente, no puede pensarse en la realidad como explicada a través de los términos de recompensa y castigo, como si el mal pudiera ser una «prueba». Es más, ¿puede hablarse de un «sentido objetivo» de las cosas? Es decir, ¿naturalmente hay bien y mal aparte de nuestras valoraciones sobre acontecimientos naturales que afectan, positiva o negativamente, nuestros intereses? 0 inos encontramos inermes en un universo del que no alcanzamos racionalmente a conocer su sentido, el sentido de la imperfección? Quizás nos empeñemos en catalogar bajo las nociones de bien y de mal hechos que no deben serlo. Porque el defecto de la «normalidad-natural»-normalidad definida desde parámetros específicos, la especie humana- quizás si pueda catalogarse como mal. El lento deterioro de un cuerpo gastado: el dolor es mal. Pero, por otro lado, «es -y ha de ser-así». Este es el problema, ¿por qué «es -y ha de ser-así»? El nacimiento y la muerte son el principio y el fin de una existencia: naturalmente son así y no hay que catalogarlos moralmente. A la naturalidad de la muerte le corresponde la del lento descenso, vertiginoso otras veces, de la vida hacia la muerte: la enfermedad... ¿Por qué es así la naturaleza? Con lo que volvemos de nuevo a la cuestión mentada: el sentido de la imperfección. ¿O quizás no haya sentido?

32 Cfr. Paniker, S., «Teología de Bangladesh», en El País, 21-V-1991. 